\title{
A Multi-Scale Energy Demand Model suggests sharing Market Risks with Intelligent Energy Cooperatives
}

\author{
Georgios Methenitis, Michael Kaisers, Han La Poutré \\ Intelligent Systems Group \\ Centrum Wiskunde \& Informatica \\ Amsterdam, The Netherlands \\ \{Georgios.Methenitis, Michael.Kaisers, Han.La.Poutre\}@cwi.nl
}

\begin{abstract}
In this paper, we propose a multi-scale model of energy demand that is consistent with observations at a macro scale, in our use-case standard load profiles for (residential) electric loads. We employ the model to study incentives to assume the risk of volatile market prices for intelligent energy cooperatives at different aggregation scales of energy consumption. Next to scale, we investigate the benefits of demand response, more precisely intelligent scheduling of time-shiftable electric processes, and virtual storage intraday and between days. Results show that the increasing electrification and introduction of flexibilities (electric vehicles, thermal applications, storage, etc.) is going to make market participation viable for smaller groups of consumers. Retailers may thus introduce innovative tariffs for intelligent energy cooperatives to share the risk of volatility in wholesale markets for electricity.
\end{abstract}

Index Terms-Cooperative systems, Demand Response, Power system economics, Smart grids

\section{INTRODUCTION}

In today's energy system, the major fraction of electricity is provided to power consumers by utility scale power producers through retail aggregators. The goal of a retailer is to buy exactly as much energy as its customers use, which can be achieved more cost-efficiently in the day-ahead market than in the intraday market. Dynamic tariffs are a means for retailers to share the risk of price fluctuations with customers. In particular, costs of planned consumption (at least a day ahead) may be priced lower than short-term deviations (procured from intraday or balancing markets). However, end-users may be repealed by the inherent uncertainty and it is an open question what market segment to address with such dynamic tariffs. In this paper we identify the minimum viable scale for assuming the risk of fluctuating prices, and show how this scale decreases by a magnitude if $10-25 \%$ of consumption are flexible in time. This suggests that retailers may offer housing cooperatives with a moderate degree of electrification and intelligent energy management innovative dynamic tariffs to share their market risks.

Obtaining a sufficiently coherent and large dataset of representative energy consumption at various scales is difficult, and may end up leading to very case-specific results. In this paper we propose a multi-scale model that captures the specific volatility and flexibility in energy demand profiles, while maintaining that the aggregate behavior is consistent with observations at a macro scale, e.g., standard load profiles (SLPs) in electricity networks. We introduce a method to derive a process aggregation model from a given SLP, which can produce synthetic demand profiles for various scales - from an individual device to a large city. Modeling of the consumption profiles will enable experimentation in simulation to study dynamic tariff viability for intelligent energy cooperatives.

The next section discusses concepts and related work in modeling electricity demand. Section III discusses the proposed multi-scale energy demand model and the required parameter derivations to make it consistent with macro observations. Section IV illustrates the resulting multi-scale energy demand and demonstrates its use by evaluating market participation of cooperatives with various scales and flexibilities. Finally Section V serves as an epilogue to this work discussing the contributions.

\section{BACKGROUND AND RELATED WORK}

Retailers must procure the power for their costumers whose energy consumption profile is not always observable, e.g., due to high cost of smart-meter roll-outs on-site. Therefore, distribution system operators (DSOs) compute standard load profiles (SLPs) based on the portfolio of end-consumers behind a measured distribution substation and for several types of consumers [1]. Example types include residential, commercial and industrial. Typically, SLPs contain an averaged daily consumption profile, based on historic data and other factors, such as the temperature. Those SLPs are used by retailers to determine the amount of energy that they must procure in the wholesale energy markets (day-ahead and intraday). Power producers sell their energy at the wholesale markets, and are influenced via the retailers by this information to plan the amount of electricity they will need to make available at any given time of the (next) day.

In order to model the quantity volatility at different scales of demand aggregation, fine-grained measurements or models are necessary. Smart-meters are electronic devices that record consumption of electric energy in small intervals of at most an hour and communicate that information at least daily back to the central system for monitoring and billing [2]. Smartmeters can gather data for remote reporting, e.g. to replace SLPs of consumer types by actual load measurements in the future. While smart-meter rollouts are in progress, the private data is usually considered sensitive, and does not generalize easily.

The unobservable consumption profiles at lower scales of aggregation, especially single household demand profiles, have given rise to different approaches to model consumption. 


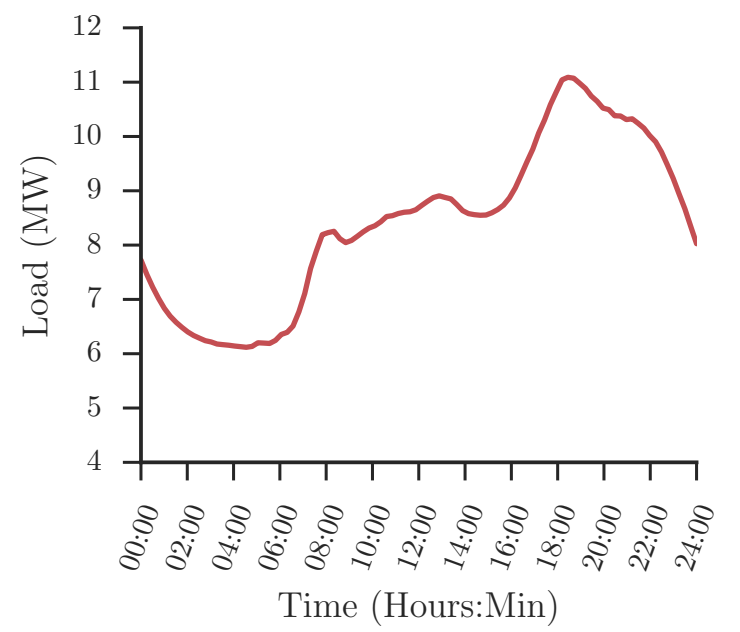

Figure 1. A typical standard load profile that captures the average household demand at the distribution substation level of aggregation, available as a public dataset [8].

A semantic approach to household consumption profiles has been studied in related work [3]. Statistics from household data are used to create a bottom-up model, approximating the consumption of a number of devices in hourly intervals. The artificial profiles generated by this model show high correlation with real data. Another model for the computation of daily electricity and hot-water demand profiles uses the mean appliance and water-tap data, as well as daylight distributions over the year [4]. The comparison between generated profiles and the end-user specific electricity measurements in a small sample of households revealed that the generated model preserved the important qualitative features of real data, which was also the case when it was applied at larger scale. While previous work in the area of generating synthetic consumption data was focused on building semantic models, we propose a universal and versatile process-model. Our model has a different focus: Rather than studying the semantics, the core focus is the multi-scale modeling. Nevertheless, our model could be extended to capture semantic dependencies, e.g. by joint distributions between the duration and consumption rate of processes. Our approach is a minimal model that can be scaled to any number of processes and can generalize to demand and supply profiles with arbitrary time horizons.

Related work has shown the impact of storage in real-time prices in relation to ramping constraints [5]; based on our model to generate synthetic load profiles, we use flexibility (storage) to study the feasibility of decreasing the number of aggregated profiles that are needed to participate directly in the wholesale market volatility. Other work has focused on typifying household load profiles into several categories [6], [7]. In a decentralized approach, different consumption profile types are important in regards to the possibility of forming groups of complementary types. Their work is thus complementary to ours.

The importance of decentralized approaches for demand side management in the future smart-grid has been shown emphasizing the improvements of the energy systems needed at the side of consumption [9]. Consumption loads can be shifted by decentralized demand side management (DSM) in the smart-grid [10]. Emergent behavior of the agents in a such a scheme alongside with the adaptation to the grid electricity price can result in peak demand reduction. This has also been studied by formulating an energy consumption scheduling game and analyzing it game theoretically [11], where agents develop strategies in regards to their daily schedules of their household appliances and loads. Best response strategies from the players resulted in a significant reduction of the peak-toaverage of the total electricity demand. While these works focus primarily on the in-depth technology specific aspects of demand response and storage, we will here perform a meta study of the merits of such technologies, independent of their implementation. Again, our primary parameter of interest is scale, but more precisely we also model storage and demand response capacities by shifting of flexible demand.

\section{METHOD}

In this section we propose the multi-scale model of energy demand and show the necessary parameter derivations to align it with macro observations, here demonstrated with standard load profiles. This model aggregates atomic individual processes, where the number of processes becomes the scaling parameter. The marginal densities of process duration, energy consumption and starting times are tuned to be coherent with macro observations, and the resulting multi-scale model can then generate synthetic data on arbitrary scales.

\section{A. Multi-Scale Model of Energy Demand}

Figure 1 presents the daily load of a distribution substation. We are interested in decomposing such a macro-scale load profile $\hat{q} \in \mathbb{R}^{n}$, where $n$ is the number of discrete time intervals (the resolution). While the proposed method is general in $n$ and could equally be applied to an annual SLP, all experiments and figures in this paper use a 24-hour time horizon with 96 quarter hourly time intervals for a consistent and intuitive presentation of the results.

1) Process Model: Every continuous (non-interrupted) consumption load incurred by a device for some duration can be considered a process. We define a process as the consumption rate $k$ that is taking place, starting at time $t_{0}$ and having a duration $\delta$. It can then be modeled mathematically as a function $f$ :

$$
\text { process: } f\left(t, t_{0}, \delta, k\right)= \begin{cases}k, & t \in\left[t_{0}, t_{0}+\delta\right) \\ 0, & \text { otherwise }\end{cases}
$$

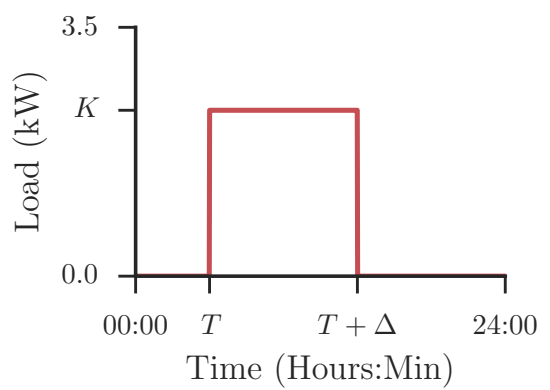

Figure 2. The multi-scale model aggregates over individual atomic processes, each characterized by consumption rate $K \mathrm{~kW}$, duration $\Delta$, and starting time $T$ 
Figure 2 presents a visual representation of the atomic process model that is characterized by consumption $K \mathrm{~kW}$, duration $\Delta$, and starting time $T$. The consumption profile of an electric device can consist of a number of processes. A typical household appliance (e.g. TV, heat pump, light bulb, electric vehicle charging, etc.) has several consumption cycles and idle functionality between these cycles, each of its consumption cycles will be treated as a separate process by the discussed model. Varying the number of processes, different aggregation levels of consumption can be approximated, from a single household appliance, to a neighborhood, or to a large city.

2) Starting Time of Process: A process has been defined as the function $f\left(t, t_{0}, \delta, k\right)$. The synthetic load profile $q_{N}$ we are interested to generate is given by the following function:

$$
q_{N}\left(t, p_{t_{0}}, p_{\delta}, p_{k}\right)=\sum_{i=1}^{N} f\left(t, t_{0}^{i}, \delta^{i}, k^{i}\right)
$$

which is the quantity of electricity of $N$ processes at timestep $t$. We assume the discrete variables $t_{0}, \delta, k$ are independent and sampled according to the discrete probability distribution functions (PDFs) $p_{t_{0}}, p_{\delta}, p_{k}$ respectively ${ }^{1}$. The expected quantity of $q_{N}$ is then equal to:

$$
\mathbb{E}\left[q_{N}\left(t, p_{t_{0}}, p_{\delta}, p_{k}\right)\right]=\sum_{N, T, K} p_{t_{0}}(T) \bar{P}_{\delta}(t-T) p_{k}(K) K
$$

where $\bar{P}_{\delta}=1-P_{\delta}$, thus $\bar{P}_{\delta}$ is the complementary cumulative density function (CCDF) of the processes' duration such that: $\bar{P}_{\delta}(t-T)=p_{\delta}(\delta \geq(t-T))$. This corresponds to the expected value of the consumption of a process at $t$ multiplied by the probability that the process started on a specific timestep $T$ and it is still active at timestep $t$ (has duration greater or equal to $t-T)$.

The interaction of $p_{t_{0}}, p_{\delta}, p_{k}$ and $\hat{q}$ has a unique solution if assumptions are made about three out of the four functions. Assuming known distributions for the PDF of a process duration $p_{\delta}$, and the consumption rate of a process $p_{k}$, we deduce the distribution over starting times from the desired macro profile $\hat{q}$. As the number of processes is increasing to infinity, the synthetic profile converges to the normalized averaged load profile $\hat{q}$ :

$$
\lim _{N \rightarrow+\infty}\left(\frac{q_{N}\left(t, p_{t_{0}}, p_{\delta}, p_{k}\right)}{N}\right)=\alpha \hat{q}(t), \alpha \in \mathbb{R}
$$

where the quantity of an infinite number of processes at timestep $t$ is approximating the normalized per process quantity of a given SLP (see Fig. 1) multiplied by a factor $\alpha$ due to the varying number of processes and the distribution $p_{k}$. We can normalize the equation, due to the law of large numbers, further saying that the expectation of $q_{N}$ is equal to a scaled version of $\hat{q}$ :

$\lim _{N \rightarrow+\infty}\left(\frac{q_{N}\left(t, p_{t_{0}}, p_{\delta}, p_{k}\right)}{N}\right)=\mathbb{E}\left[\frac{q_{N}\left(t, p_{t_{0}}, p_{\delta}, p_{k}\right)}{N}\right]=\alpha \hat{q}(t)$

which is the expected quantity per process at timestep $t$.

\footnotetext{
${ }^{1}$ A straight-forward modification allows to treat the variable $k$ as continuous, while making $t_{0}$ and $\delta$ continuous requires more careful adaptation of the model, which may not have a unique solution in that case.
}

Using equation (3) we can infer the PDF of $t_{0} \in[0, n]$ where $n$ is the time horizon and depends on the chosen resolution for the time axis. We start from the expected value of $q_{N}$ at timestep $t$, due to independence we can rewrite the equation as:

$$
\mathbb{E}\left[q_{N}\left(t, p_{t_{0}}, p_{\delta}, p_{k}\right)\right]=N \mathbb{E}[k] \sum_{T} p_{t_{0}}(T) \bar{P}_{\delta}(t-T)
$$

replacing the first term according to equation (5) we have:

$$
\frac{\alpha}{N \mathbb{E}[k]} \hat{q}(t)=\hat{q}^{\prime}(t)=\sum_{T} p_{t_{0}}(T) \bar{P}_{\delta}(t-T)
$$

From equation (7) we can produce a set of linear equations which form the linear system of the form $A x=b$ :

$$
\left[\begin{array}{cccc}
\bar{P}_{\delta}(0) & \bar{P}_{\delta}(n) & \ldots & \bar{P}_{\delta}(1) \\
\bar{P}_{\delta}(1) & \bar{P}_{\delta}(0) & \ldots & \bar{P}_{\delta}(2) \\
\vdots & \vdots & \ddots & \vdots \\
\bar{P}_{\delta}(n) & \bar{P}_{\delta}(n-1) & \ldots & \bar{P}_{\delta}(0)
\end{array}\right] \times\left[\begin{array}{c}
p_{t_{0}}(0) \\
p_{t_{0}}(1) \\
\vdots \\
p_{t_{0}}(n)
\end{array}\right]=\left[\begin{array}{c}
\hat{q}^{\prime}(0) \\
\hat{q}^{\prime}(1) \\
\vdots \\
\hat{q}^{\prime}(n)
\end{array}\right]
$$

Normally matrix $A$ would be a lower triangular matrix, where processes that influence the probability density at timestep $t$ are only the ones starting at timesteps $t_{0} \in[0, t]$. In order to model the continuity of our model over the time horizon we assume wrap-around, i.e. processes which start late in the day and have a duration that lasts longer than the day's horizon influence the expected quantity at the beginning of the same day (or whatever the horizon of the profile). In this way, matrix $A$ models the past and the future processes that may affect the current PDF at timestep $t$. Equation (7) becomes:

$$
\hat{q}^{\prime}(t)=\sum_{T} p_{t_{0}}(T) \bar{P}_{\delta}(s), s= \begin{cases}t-T & , \forall T \in[0, t] \\ n-|t-T|, \forall T \in(t, n]\end{cases}
$$

Looking at the first row of matrix $A$ that contains the probabilities for every timestep for the duration of a process that starts at timestep 0 , we can infer that the expected quantity for $q$ will be the probability of a process starting at timestep zero and having at least 0 duration, starting at timestep 1 and have duration at least $n$, or starting at timestep $n$ and having duration at least 1. Solving the linear system will result in obtaining the scaled distribution of $p_{t_{0}}$ due to the scaled quantity $\hat{q}^{\prime}$. Normalizing $p_{t_{0}}$ to sum up to one yields the PDF of the process starting times. The inferred probabilities $p_{t_{0}}$ can be used in equation (2) to generate synthetic load profiles.
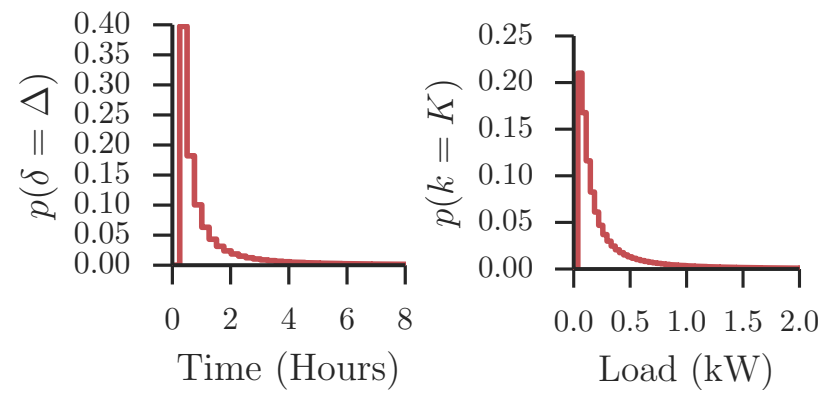

Figure 3. Left: Probability density function $p_{\delta}$ for the duration (hours) of processes (shown for duration up to 8 hours). Right: Probability density function $p_{k}$ over consumption rates $(\mathrm{kW})$ of the processes (shown for load up to $2 \mathrm{~kW}$ ). 


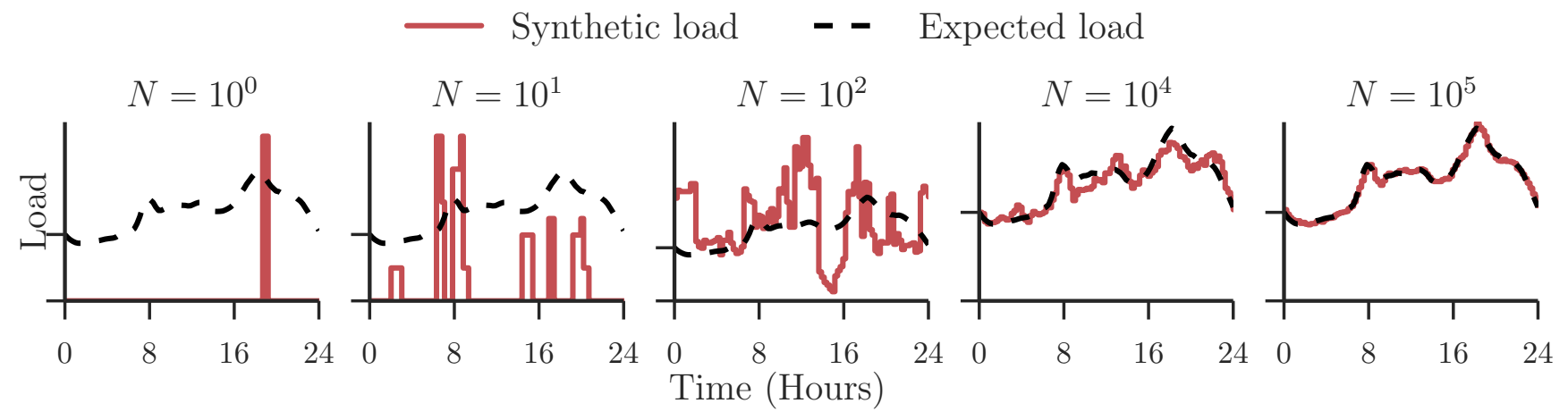

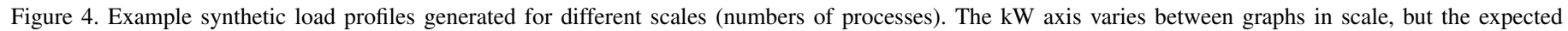
quantity is given for comparison. The synthetic profile is approximating the expected value as the number of processes increases.

\section{EXPERIMENTS AND RESULTS}

We start this section by showing the way synthetic load profiles can be generated using the method in the previous section. The resulting load profiles are the basis for scaling experiments, studying the price of electricity in market participation under varying assumptions of flexibility.

\section{A. Multi-Scale Model of Electricity Demand}

The algorithm, described in detail in Section III-A takes as input the SLP $\hat{q}$ (see Fig. 1), the number of processes $N$ and the PDFs for the duration and the consumption rate of a process, $p_{\delta}$ and $p_{k}$ respectively, given in Figure 3 . The resulting multiscale aggregation model of processes is illustrated in Figure 4. All discrete signals used for the quantities described in this section have 96-values, using 15-minute intervals throughout a day. The PDFs $p_{\delta}, p_{k}, p_{t_{0}}$ have the same resolution. In general, the model does not put any restrictions on the distributions that can be used. In this experimental setup, we chose independent F-distributions (heavy-tailed) for both PDFs, based on consumption statistics for household appliances [12]. Since we are looking into the daily consumption profile of a number of processes, we truncate the duration probability function from 0 to 24 hours to capture the whole margin of a day. The consumption distribution is from zero to $3.68 \mathrm{~kW}$, given that the maximum electric flow of household connections are typically $16 \mathrm{~A}(230 \mathrm{~V})$.

Figure 3, presents the PDFs for both the consumption rate and the duration processes. Given the two distributions, the model can be obtained solving the linear system (8), the result of the linear system of equations when normalized to sum up to one is the probability density function of the starting time of a process $p_{t_{0}}$. Figure 5 illustrates the distribution of probability for the starting time of a process over the day time horizon. Notice that the probability that a process starts is highly increasing after 6 am, and increasing again after 5 pm when people get up or come home and tend to turn on many devices.

Given the PDF of the starting times $p_{t_{0}}$ we can generate synthetic load profiles for specific scales $N$. Recall equation (4): as the number of processes increases, the synthetic profile that is obtained approximates the expected load $\mathbb{E}[q]$. As expected, Figure 4 shows how using the derived $p_{t_{0}}$ in equation (9) indeed gives rise to synthetic profiles that approximate the SLP $\hat{q}$ for large $N$. A single device with expected daily consumption of $2.3 \mathrm{kWh}$ can be approximated when $N=10^{1}$. For $N=10^{2}$ the profile resembles the consumption of a household (23 kWh daily consumption). An aggregation of the consumption profiles of several apartments in a building is close to $N=10^{3}$ (230 $\mathrm{kWh}$ daily consumption). A low voltage transformer with up to a hundred households corresponds to $N=10^{4}$. The measurement of the consumption at the substation ( $240 \mathrm{MWh}$ daily load, $N=10^{6}$ ) is very close to the expected quantity. We chose to show a smaller substation scale $\left(N=10^{5}\right)$, because this already yields very smooth behavior.

\section{B. Balancing Incentives from Sharing Market Risk}

In the future smart-grid, small collectives of households may participate as entities in the wholesale market volatility instead of paying flat tariffs for their daily consumption. This would be a vital contribution to tapping into local flexibilities, which are thus far unavailable to the market, since retail customers have no financial incentives to align their behavior with the power grid's needs. We perform two experiments, studying the price per $\mathrm{kWh}$ that can be achieved at different scales and with varying flexibility, which is first provided

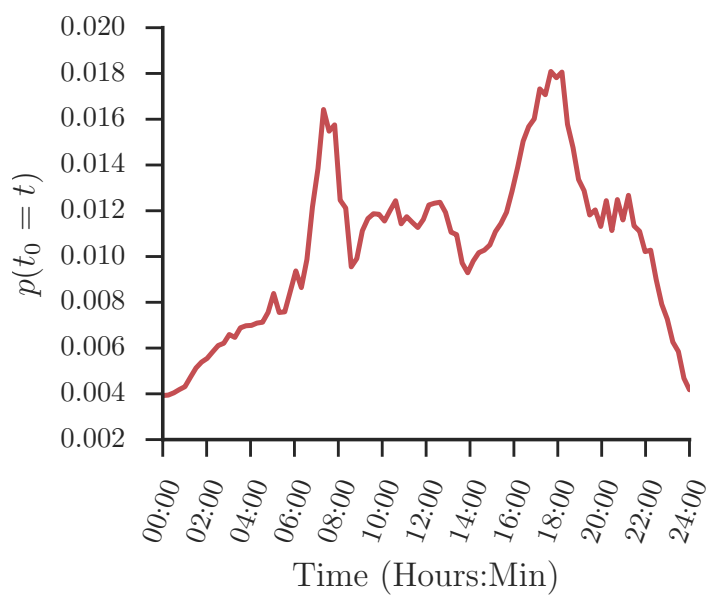

Figure 5 . The probability density function $p_{t_{0}}$ characterizes the starting times of the processes, and is shown as derived from the standard load profile in Figure 1, and marginal densities in Figure 3. 


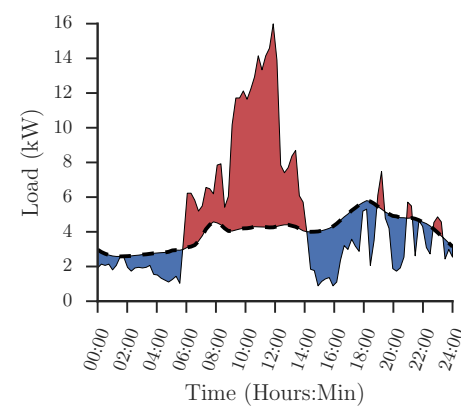

(a) Virtual storage

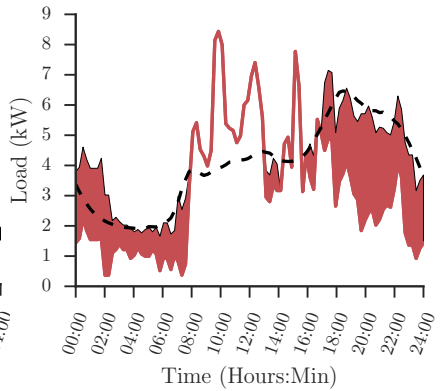

(b) Shiftable processes
Figure 6. The dashed line indicates the power profile procured on the dayahead market. (a): the shaded areas indicate the actual shortage (above) and excess capacity (below). Virtual storage applies a daily netting of shortage and excess, and provides an optimistic bound on any (possibly lossful) storage (b): solid line shows power consumed by uncontrollable processes, and the shaded area indicates power consumed by time-shiftable processes, placed with a sequential greedy heuristic.

by a virtual battery and second by demand response, more specifically by time-shifting flexible processes. For the retail baseline, we assume a flat electricity tariff of $0.22 € / \mathrm{kWh}$. A cooperative participating in the wholesale market volatility may buy either on the day-ahead market, where prices are more formidable but information is limited to the anticipated expected demand $\hat{q}$ scaled according to $N$, or on the day itself via balancing markets (in practice either the intraday market or going into imbalance, which is automatically settled by the DSO through reserve markets). We assume a $0.15 € / \mathrm{kWh}$ price for the day-ahead market, adding taxes and surcharges to the trading price but saving the retailer risk margin when assuming the market risk itself. For the balancing we assume a 10 -fold penalty cost $(1.50 € / \mathrm{kWh})$ for energy that has not been procured on the day-ahead market. Over-procured capacity is wasted without additional cost.

1) Virtual Storage: Flexibility is modeled as the percentage of the daily consumption that can be transferred within the day, resulting in the reduction of imbalances to be procured on the balancing market. As an optimistic scenario (lower bound of costs) of any intraday flexibility, we assume lossless storage in the form of a daily netting (virtual battery), as illustrated in Figure 6a. The results are given in Figure 7a, which presents the price per $\mathrm{kWh}$ for four levels of flexibility over various scales. For comparison, the retail and ahead market prices are given. Market participation without flexibility is viable from a magnitude of $10^{4}$ processes, which is the crucial point when a cooperative can assume the risk of wholesale market price volatility as the achieved total costs are getting smaller than the flat retail market tariffs. Lossless storage of just $10 \%$ would move the minimum viable scale down by a magnitude, with further flexibility having lesser effect.

2) Intelligent Demand Response: We now use a more sophisticated method to model the flexibility induced by demand response in the future smart-grid, illustrated in Figure 6b. Demand response is modeled as (a subset of) time-shiftable processes. We use a fraction (e.g., 10\%) of the total number of processes as processes that can be moved during the day. However, the processes are not know on the day ahead. This corresponds to the assumption that it may be unclear how empty the car battery will be, or how big a laundry needs to be run. Thus, even time-shiftable processes remain stochastic in the two variables of consumption and duration. Having a number of processes that can start at any time during the day, the agent uses an intelligent (sequential, greedy) heuristic to choose the best starting time that each of them will take. For each time-shiftable process, the starting time that minimizes positive imbalances is chosen sequentially, considering the sum of all uncontrollable and previously placed processes, until all processes are placed one by one. Figure $6 \mathrm{~b}$ presents a consumption profile of 200 processes from which $40(20 \%)$ of them have been shifted. The same prices are used in this experimental setup as the ones introduced in the previous experiment. Figure $7 \mathrm{~b}$ presents the price per $\mathrm{kWh}$ that is obtained at different scales in the wholesale and retail market with or without time-shiftable processes. The non-flexible line is equivalent to the previous experiment, while demand response flexibility is more restricted than the virtual battery due to the continuation of processes. Similar to the previous experiment, flexibility decreases viable market participation scale by a magnitude. However, at least $25 \%$ demand response are needed to achieve this result. The large price increase at lower scales is primarily attributed to the unpredictable total energy consumption during the trading horizon of a full day. This implies that demand curtailment, and shifting between consecutive trading days provides a key resource to further decrease the viable scale of electricity market volatility participation.

3) Between-Day Storage: While the previous two case studies focused on the benefits of intraday demand response, here we extend the first model of virtual storage by incorporating between-day transfer. We assume each day starts with a reserve capacity (e.g. battery or heat buffer) equal to the flexibility percentage of the actual daily consumption. During the day, shortages of electricity are balanced using demand response (see Figure 6a). In addition, the total energy consumption that day can be curtailed using the between-day storage. Replenishing this storage is planned for the next day with the day-ahead price. While we retain the lossless model for intraday demand response (as a model of time-shifting), the balancing quantity for recharging in the next day is penalized by a loss factor of $30 \%$ - this corresponds to typical roundtrip storage losses in a battery or heat buffer, and increases the quantity bought the next day. Comparable to previous results, Figure 7c presents the price per kWh for four different percentages of flexibility and for varying scales. Note that betweenday transfer yields high gains despite the losses involved in medium-term storage. If storage capacity exceeds average daily consumption, results show that participation in the wholesale market volatility becomes viable for an aggregation level of 100 processes (a large household), an order of magnitude less than using mere intraday demand response.

\section{CONClusions}

We have introduced a multi-scale energy demand model, aggregating processes, and an algorithm for alignment with given macro observations, such as SLPs. Experiments show the business case for participating in the wholesale electricity market volatility at varying aggregation scales, and how flexibility in the form of (virtual) storage and demand response 


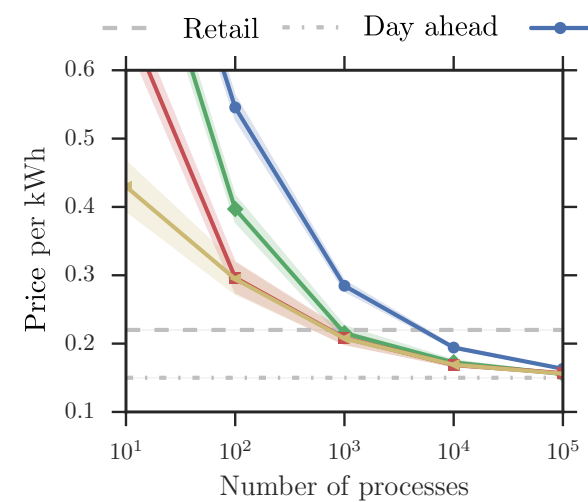

(a) Virtual storage

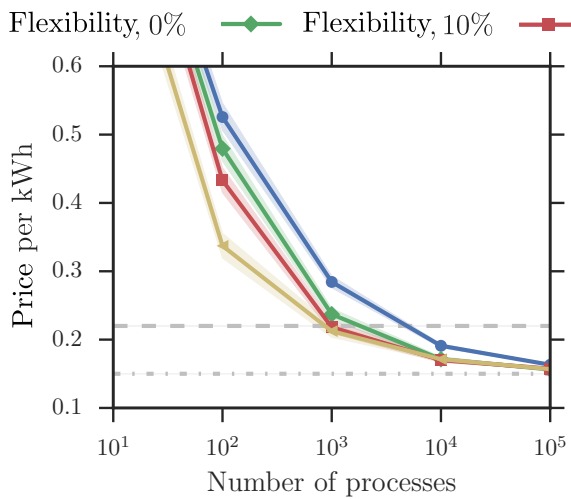

(b) Intelligent demand-response

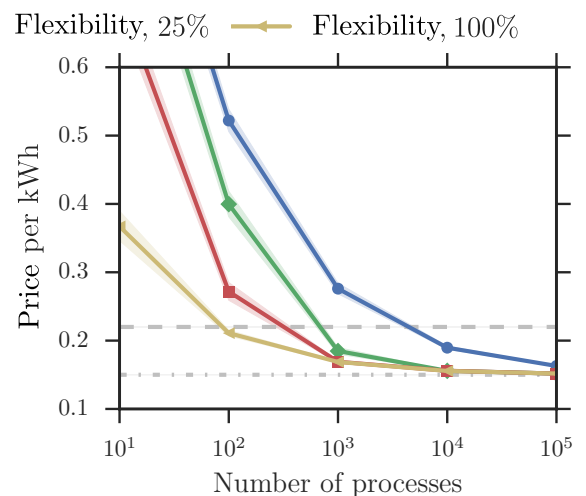

(c) Between-day storage

Figure 7. Electricity price (Euro) per $\mathrm{kWh}(€ / \mathrm{kWh})$ for different aggregation scales and four levels of storage capacities. Mean values computed using $200-$ independent samples, $95 \%$ confidence interval is shown by the shaded areas.

reduces the minimum viable scale. The decrease in costs given sufficient flexibility, especially between-day, may bring the required aggregation scale down to the size of common place existing housing cooperatives, such as large apartment buildings. These cooperatives often already make joint longterm investments into their infrastructure, and are potential customers for dynamic tariffs. These tariffs provide an incentive to invest in increasing flexibilities (electric vehicles, HVAC, etc.) to enable the consumers to assume and mitigate risks of increased costs of unplanned consumption, or to increase self-consumption and thus balancing of local (e.g. solar) generation.

For the sake of clarity and brevity, upfront investment costs and monthly fixed costs have been neglected, and other simplifying assumptions have been made. First, the duration, power consumption and starting time of processes were treated as independent variables. Second, only one type of atomic process has been used. Third, only one type of (residential) consumption has been approximated. The plasticity of our model allows straight-forward extensions to address each of these limitations. These extensions include but are not limited to (1) joint distributions over process characteristics, (2) considering further process types (e.g., recurring cycles), (3) approximating other demand types, supply profiles (e.g., of solar or wind generation), or even other commodities (heat, gas). In addition, the ahead-market has been tackled heuristically and can be optimized to further improve the gains. The proposed model is therefore more widely applicable to study multi-scale effects of demand and supply aggregation and economics of flexibilities in energy systems.

\section{REFERENCES}

[1] J. A. Jardini, C. Tahan, M. Gouvea, S. U. Ahn, and F. Figueiredo, "Daily load profiles for residential, commercial and industrial low voltage consumers," Power Delivery, IEEE Transactions on, vol. 15, no. 1, pp. 375-380, 2000.
[2] S. S. S. R. Depuru, L. Wang, and V. Devabhaktuni, "Smart meters for power grid: Challenges, issues, advantages and status," Renewable and sustainable energy reviews, vol. 15, no. 6, pp. 2736-2742, 2011.

[3] J. V. Paatero and P. D. Lund, "A model for generating household electricity load profiles," International journal of energy research, vol. 30, no. 5, pp. 273-290, 2006.

[4] J. Widén, M. Lundh, I. Vassileva, E. Dahlquist, K. Ellegård, and E. Wäckelgård, "Constructing load profiles for household electricity and hot water from time-use datamodelling approach and validation," Energy and Buildings, vol. 41, no. 7, pp. 753-768, 2009.

[5] N. Gast, J.-Y. Le Boudec, A. Proutière, and D.-C. Tomozei, "Impact of storage on the efficiency and prices in real-time electricity markets," in Proceedings of the fourth international conference on Future energy systems. ACM, 2013, pp. 15-26.

[6] D.-W.-I. C. Flath, D.-W.-I. D. Nicolay, T. Conte, P. D. C. van Dinther, and L. Filipova-Neumann, "Cluster analysis of smart metering data," Business \& Information Systems Engineering, vol. 4, no. 1, pp. 31-39, 2012.

[7] M. Hayn, V. Bertsch, and W. Fichtner, "Electricity load profiles in europe: The importance of household segmentation," Energy Research \& Social Science, vol. 3, pp. 30-45, 2014.

[8] Retail Market Design Service (RMDS). (2015, May) Standard load profiles developed for the irish market. [Online]. Available: http://rmdservice.com/standard-load-profiles/

[9] P. Palensky and D. Dietrich, "Demand side management: Demand response, intelligent energy systems, and smart loads," Industrial Informatics, IEEE Transactions on, vol. 7, no. 3, pp. 381-388, 2011.

[10] S. D. Ramchurn, P. Vytelingum, A. Rogers, and N. Jennings, "AgentBased Control for Decentralised Demand Side Management in the Smart Grid," pp. 5-12, 2011.

[11] A. H. Mohsenian-Rad, V. W. S. Wong, J. Jatskevich, R. Schober, and A. Leon-Garcia, "Autonomous demand-side management based on game-theoretic energy consumption scheduling for the future smart grid," IEEE Transactions on Smart Grid, vol. 1, no. 3, pp. 320-331, 2010.

[12] J.-P. Zimmermann, M. Evans, J. Griggs, N. King, L. Harding, P. Roberts, and C. Evans, "Household electricity survey: A study of domestic electrical product usage," Intertek Testing \& Certification Ltd, 2012. 